\title{
Integrating High Order Numerical Techniques and Magnetic Resonance Imaging for Fluid Flow Investigation
}

\author{
BRANCOLINI, Alberto, Agip SpA \\ COMINELLI, Alberto, Agip SpA \\ CONSONNI, Pietro, Agip SpA \\ MANTICA, Stefano, Agip SpA \\ RADAELLI, Francesco, Agip SpA
}

Paper presented at the 5th European Conference on the Mathematics of Oil Recovery, Leoben, Austria, 3-6 Sept. 1996

\begin{abstract}
In this paper we present an integrated approach, via sophisticated experimental and numerical techniques, to the investigation of displacement phenomena in porous media. A non-destructive imaging technique, Magnetic Resonance Imaging (MRI), has been used to study fluid flow in core plugs, and a Mixed Finite Element/TVD Finite Volume simulator to reproduce the observed data. The main advantage of the MFE/FV code is its capability of accurately solving physical phenomena without introducing unrealistic numerical dispersion or oscillations. Comparison with standard Finite Differences (FD) code has been performed.
\end{abstract}

\section{INTRODUCTION}

Investigation and simulation of the flow of two or more fluids in porous media is a key issue in the oil industry in order to improve reservoir performance.

Until the introduction of non-destructive imaging techniques (Magnetic Resonance Imaging, CT X-ray) the observation of fluid distribution in rock and its evolution as a function of time could only be done indirectly. Conventional methods provide only the average of such properties as porosity, saturation and permeability.

There have been a number of applications of MRI in the study of flow dynamics and porosity on laboratory-size rock plugs (Ref.1). This paper presents the results of using MRI to complement "room condition" core flood test instrumentation to measure saturation distribution inside core plugs using one- and two-dimensional profile images.

In one-dimensional profile images, a drainage and two imbibition experiments were carried out with an unsteady-state approach, which is often a preferable way of conducting displacement tests which simulate a reservoir process. In the two-dimensional case, deuterium water was injected into a water saturated sample.

All the different experiments were also simulated using both a traditional finite difference simulator and a mixed finite-elements (MFE) total variation diminishing (TVD) finite-volume (FV) code.

Mixed finite-elements methods have proven to give high order accurate approximations of both velocity and pressure over general unstructured grids (Ref. 2). At the same time, TVD schemes guarantee solutions free of numerical diffusion and spurious oscillations near sharp fronts (Ref.3)

Moreover, the coupling MFE-TVD FV is optimal in the sense that the fluxes which are primary variables for the pressure equation are directly used in the FV solver.

The approach proposed in this paper makes it possible not only to check the reliability of different numerical schemes, but also to separate the contribution of the single forces presents during the displacements (mainly capillary and viscous), from the unphysical numerical approximation.

\section{NUCLEAR MAGNETIC RESONANCE (NMR) PRINCIPLES}

Nuclear Magnetic Resonance as a spectroscopic technique serves as a tool for qualitative and quantitative structural analysis. It involves all the atomic nuclei possessing spin. Spin is the quantum mechanical property that is responsible for bulk magnetic effects. Nuclei placed in a magnetic field, $\mathrm{B}_{0}$ along, for example, the $\mathrm{z}$ direction, give a macroscopic magnetisation parallel to the magnetic field (Ref.4). Without this external field the spins are oriented randomly.

In fact these nuclei are distributed over discrete energy levels with an energy difference between them equal to 


$$
\mathrm{E}=\frac{\mathrm{h}}{2 \pi} * v
$$

where $h$ is the Plank constant, and $v$ is the frequency of resonance or Larmor frequency.

At equilibrium, the low energy level is the most populated, giving the system a magnetisation parallel to the magnetic field.

The thermal energy tends to equalise the spin alignment along and against $\mathrm{B}_{0}$, i.e. it tends to produce the same population between energy levels, giving a final difference of spin population described by the Boltzman distribution

$$
\propto \exp \left(\frac{\Delta E}{k T}\right)
$$

where $\mathrm{k}$ is the Boltzman constant, $\mathrm{T}$ the temperature of the system and $\Delta \mathrm{E}$ is the energy difference between spin levels.

The energy difference is measured by a resonance method (i.e. energy at just the right frequency, the frequency of resonance, is applied and the relative adsorption recorded). In addition to the main field, the sample is subjected to a second oscillating field $B_{1}$, perpendicular to $B_{0}$, with a frequency equal to the Larmor one defined as

$$
v=\gamma^{*} \mathrm{~B}_{0}
$$

where $\gamma$ is the gyromagnetic ratio characteristic of the nucleus.

The nuclei distribution over the different levels will change, producing a change in magnetisation.

In the course of the NMR experiment, the net magnetisation vector may be tipped away from the $B_{0}$ direction by 180 degrees, 90 degrees or some fraction thereof. After this, a signal is observed because when $B_{1}$ has been switched off, the precession of the magnetic moment induces a voltage in the receiver coil situated in the $x$-y plane. When the net magnetisation vector is tipped into the $x-y$ plane and detected, the signal obtained is called free induction decay (FID). The return to equilibrium is governed by relaxation mechanisms and two different types can be distinguished:

1) Spin-lattice or longitudinal relaxation. It describes the return of magnetisation along the $z$ direction. The typical time is indicated as $T_{1}$

2) Spin-spin or transverse relaxation. It describes the decay to zero of the magnetisation in the $x-y$ plane. The characteristic time is indicated as $\mathrm{T}_{2}$.

Until now we have thought of the sample as being in a homogeneous field. For imaging purposes, however, we have to apply a gradient superimposed on the $B_{0}$ field to spatially encode the signal. In fact, if we apply a field gradient, for example along the $\mathrm{x}$ direction, the $\mathrm{y}-\mathrm{z}$ plane will be divided into columns each at different field and with different frequencies of resonance. So we can recognise from which part of the plane the signal comes.
In the same way, two gradients along two different directions make it possible to encode the signal from a pixel.

\section{IMAGING PROCEDURE AND EXPERIMENTAL DETAILS}

Dynamic displacement experiments were conducted using two different Berea Sandstone samples and one real reservoir rock. All the Berea samples had $5.08 \mathrm{~cm}$ diameters and $6.85 \mathrm{~cm}$ lengths while the field sample was $6.6 \mathrm{~cm}$ and $10 \mathrm{~cm}$ respectively. They were sealed with resin (ARALDIT RESIN CW2215 ${ }^{\circledR}$, CIBA-GEIGY) and two caps made of teflon were used to connect the sample with the flow line. TAB. 1 reports the petrophysical characteristics of the plugs and the various experimental conditions.

The fluids used were water, air, deuterium water and perfluorinated hydrocarbon $\left(\right.$ GALDEN $^{\circledR}$ HT135, MONTEFLUOS).

Deuterium water $\left(\mathrm{D}_{2} \mathrm{O}\right)$ has the same characteristics as water but doesn't present an NMR signal at the frequency of resonance of the ${ }^{1} \mathrm{H}$.

Perfluorinated fluid is a hydrocarbon where the hydrogen has been substituted with ${ }^{19} \mathrm{~F}$. In this way, it is possible to follow the water phase using ${ }^{1} \mathrm{H}$ or the oil phase with ${ }^{19} \mathrm{~F}$. These two fluids are typically used as doped fluids and they make it possible to follow the movement of only one phase in each experiment. For example, during an oilwater displacement where perfluorinated fluid is used and with the NMR acquisition parameters set to the frequency of resonance of the ${ }^{1} \mathrm{H}$, the only phase visible will be the water.

MRI imaging was performed with a BRUKER BIOSPEC $24 / 40$ equipped with a $40 \mathrm{~cm}$ bore superconducting magnet, with gradient strength up to $50 \mathrm{mT} / \mathrm{m}$. A multislice and multi-echo sequence was applied to acquire the 1D profile and $2 \mathrm{D}$ image, with some modifications in the 1D application in order to reduce the echo time (TE). This sequence is illustrated in FIG 1.

To acquire the multi-echo longitudinal ID profile, both the slice and the phase encoding are eliminated from the sequence. We used 6-10 different echo times (TE), depending on the experiment. The 1D profile was obtained acquiring a series of single (Hahn) echoes, increasing the TE from one experiment to the next, with a delay (TR) between each echo acquisition greater than $5 T_{1}$. This delay ensures that magnetisation $\left(M_{0}\right)$ has fully recovered between echo. The echoes so acquired consent an extrapolation at the initial time of the real magnetisation, without any relaxation effect.

The 2D images were also acquired with 6-10 echo times, depending on the experiment. The ID profile thus represents the total projection of the sample on a longitudinal plane and it can represent the frontal displacement during the dynamic experiment. The 2D 
image of a thin slice along the longitudinal plane of the sample can describe the eventual presence of fingering or the level of stability of the displacement.

The 2D images at different TEs were the result of normal multi-echo sequences.

In order to quantify, in terms of saturation values, the acquired profiles obtained from the different NMR acquisitions, as mentioned before, it is necessary to extrapolate the magnetisation intensities to the initial time $(t=0)$. In fact the effect of the relaxation processes described by the two times $T_{1}, T_{2}$ is to reduce the real magnetisation before detection can start. At the same time the knowledge of the relaxation functions and parameters allows an estimation to be made of the real value fitting the experimental data. In our experiment the relaxation mechanism can be described as follows:

$$
M(t)=M(0) * \exp \left(t / T_{1}\right)
$$

The decay behaviour is not, however, simply described by a mono-exponential function but needs a multiexponential approach (Ref 5,6). For our purposes it was enough to use a bi-exponential function of the form (4) to obtain an error less than $10 \%$ on the saturation.

Gravimetric analysis and integration under the profile were used to convert the observed local magnetisation into local saturation across each plug.

\section{NUMERICAL SIMULATION}

The two main sources of error in finite difference methods, employed for reservoir simulation, occur in the approximation of the fluid pressure and velocity, and in the technique of upstream weighting adopted to stabilise the conservation equation.

Velocity variables are usually obtained from Darcy's law via a process of numerical differentiation of the pressure and multiplication by the discontinuous permeability function $\mathbf{K}$. In general, this process does not give accurate and local mass conservative schemes. Moreover, steep fronts are not well resolved by upstream weighting due to the inherent numerical diffusion implicit in this technique. In this paper we present numerical simulations involving two phase flow in one or two-dimensional scenarios. In either case we have employed a full 2D compressible code based on a splitting of equations and on advanced approximation techniques.

Firstly, a parabolic equation for the pressure and a convection-dominated conservation law for the phase saturation are developed, following the scheme proposed by Trangenstein and Bell (Ref.7).

High order mixed finite element and Godunov finite volume methods are then employed for the discretization of the resulting pressure and component conservation equations, respectively.

\section{THE PRESSURE AND VELOCITY EQUATIONS}

In the following we shall limit our investigation to the case of a slightly compressible wetting(w)-non-wetting(n) system in the absence of gravitational contribution terms. The parabolic equation for the pressure $p$ of the nonwetting phase is then derived from a linearization of the conservation of volumes (Ref.8).

$$
\Phi C_{\mathrm{T}} \frac{\partial \mathrm{p}}{\partial \mathrm{t}}+\vec{\nabla} \cdot\left(\mathrm{f}_{\mathrm{n}} \overrightarrow{\mathrm{v}}_{\mathrm{t}}\right)=\mathrm{g}
$$

where $\phi$ is the porosity of the medium, $\mathrm{C}_{\mathrm{T}}$ is the total compressibility of the system (fluid plus rock), $f_{n}$ is the non-wetting phase fractional flow and $v_{t}$ is the total velocity: $v_{w}+v_{n}$. Using Darcy's law:

$$
\lambda_{\mathrm{t}}^{-1} \overrightarrow{\mathrm{v}}_{\mathrm{t}}=-\vec{\nabla} \mathrm{p}-\lambda_{\mathrm{n}} \vec{\nabla} \mathrm{p}_{\mathrm{nw}}
$$

(with $\lambda_{n}$ mobility of non-wetting phase and $p_{n w}$ capillary pressure term), in a MFE framework, the second order equation for pressure is rewritten, formally, as a first order system in the pressure and velocity unknowns.

We have adopted here the lowest order MFE scheme presented by Brezzi-Douglas-Marini [BDM_1] (Ref.9) which entails the approximation of pressure as piecewise constant over each element and velocity, associated to element edges, as piecewise linear:

$$
\begin{aligned}
& \vec{v}=\sum_{i=1}^{2 L} v_{i} \vec{\psi}_{i} \ldots \ldots \ldots \ldots \ldots \ldots \ldots(7) \\
& \mathrm{p}=\sum_{j=1}^{M} \mathrm{p}_{j} \omega_{j} \ldots \ldots \ldots \ldots \ldots \ldots \ldots . . . \ldots \ldots \ldots
\end{aligned}
$$

where $L$ represents the total number of edges and $M$ the total number of elements, $v_{i}, p_{j}, t_{l}$ and $\omega_{j}$ the velocity and pressure degrees of freedom and basis functions, respectively.

As shown in a previous work (Ref.10), despite the larger number of variables involved, this space is computationally more efficient than the usual lowest order Raviart-Thomas space $\mathrm{RT}_{0}$. Furthermore, this coupling gives second order accurate solutions for both pressure and velocities.

After projection of the equations onto the discretization spaces, with some manipulations (see Ref.8 for details) we obtain an algebraic linear system:

$$
\left(\begin{array}{cc}
\mathrm{A} & \mathrm{C} \\
\mathrm{C}^{\mathrm{T}} & -\mathrm{D}
\end{array}\right)\left(\begin{array}{l}
\mathrm{v} \\
\mathrm{p}
\end{array}\right)=\left(\begin{array}{l}
\mathrm{r}_{1} \\
\mathrm{r}_{2}
\end{array}\right) .
$$

where $C$ is the discretization of the divergence operator, $\mathrm{A}$ and $\mathrm{D}$ are the velocity and pressure stiffness matrix, respectively.

We solve this system via a Schur complement decomposition w.r.t. $\mathrm{D}$ of the full matrix which restate the problem as a symmetric positive definite one. 


\section{THE CONSERVATION LAWS}

In the sequential approach considered in this paper a conservation law is derived for the saturation of the nonwetting phase. Denoting the advective and diffusive flux respectively as $F_{\text {adv }}$ and $F_{\text {dif }}$, we can write:

$$
\frac{\partial \mathrm{s}}{\partial \mathrm{t}}+\vec{\nabla} \cdot\left(\overrightarrow{\mathrm{F}}_{\mathrm{adv}}+\overrightarrow{\mathrm{F}}_{\mathrm{dif}}\right)=0 \ldots \ldots \ldots . . .(10
$$

where $\mathrm{s}$ is the non-wetting phase saturation.

The diffusive flux can be written as a function of saturation and capillary pressure:

$$
\overrightarrow{\mathrm{F}}_{\mathrm{dif}}=\lambda_{\mathrm{w}} \frac{\partial \mathrm{p}_{\mathrm{nw}}}{\partial \mathrm{s}} \vec{\nabla} \mathrm{s}
$$

Given a sequence of discrete time levels $t^{i}$, we apply operator splitting to (10) first solving:

$$
\frac{\partial \overline{\mathrm{s}}}{\partial \mathrm{t}}+\vec{\nabla} \cdot \overrightarrow{\mathrm{F}}_{\mathrm{adv}}(\mathrm{x}, \mathrm{t}, \overline{\mathrm{s}})=0 \ldots \ldots \ldots \ldots
$$

with initial condition $\mathbf{s}^{\mathrm{n}-1}$.

Denoting by s" the solution of (12) we next solve:

$$
\frac{\partial \mathrm{s}}{\partial \mathrm{t}}+\vec{\nabla} \cdot \overrightarrow{\mathrm{F}}_{\mathrm{dif}}(\mathrm{x}, \mathrm{t}, \mathrm{s})=0
$$

with initial condition $s^{*}$. The solution generated will approximate $\mathrm{s}^{\mathrm{n}}$ (Ref.11).

In general, (12) is a non strictly hyperbolic equation and can be solved with the methods presented by Bell et al. (Ref.12) and applied in (Ref.8) to black oil simulations.

Next, we have discretized equation (13) via a MFE-RT $T_{0}$ scheme and an implicit Euler time approach in order to directly approximate the flux function and allow large time steps.

\section{RESULTS AND DISCUSSION}

Three different experiments were carried out on two Berea sandstones. For these samples the relative permeability and capillary pressure curves were widely studied and we obtained them from the literature (Ref.13, 14)

For each experiment a set of dimensionless flow parameters were computed in order to define the dominant forces (Ref.15, 16). TAB. 2 shows the values of the scaling parameters.

The first was an imbibition flooding of water in a water wet sample at irreducible saturation. The scaling parameters indicate a capillary regime which is confirmed by the experimental front shown in FIG 2. In fact a spread of the water profile in the direction of the output face is quite evident. In the picture the experimental and numerical water fronts are shown at different injection times: initial state, 25, 37 and 47 minutes. Both $\mathrm{FD}$ and MFE-TVD FV simulators well describe the water advance because the displacement is capillary dominated (TAB.2). Consequently, the numerical dispersion effect is covered by the physical diffusion. Experimental front at time 47 minutes shows a clear end-effect that is not described by the two simulators. However, it is a typical core effect which does not influence field scale simulation.

The second imbibition experiment differs from the previous one in respect of a flow rate which is 10 times higher. Also the irreducible water saturation has moved from an average value of $22 \%$ to $14 \%$. From TAB.2 this case appears in a transition zone between purely capillary flow and viscous flow. In FIG.3 sharper water fronts are shown, after 2 and 4 injection minutes. The comparison between the two simulators reveals the effect of the numerical dispersion. In fact, while MFE-TVD FV simulation captures in a correct way the shape of the experimental front for all the saturation values, FD simulation appears to be unable to match it accurately, especially at low water saturation values.

We wish, again, to underline that both experiment 1 and 2 have been simulated using the same set of capillary pressures, obtained by tuning the values derived from literature (Ref.13). To get a correct match, we also had to reduce water-relative permeability for experiment 1 by a constant factor with respect to literature values (Ref.14), while we used the original values for experiment 2 . This assumption is justified by rate and irreducible saturation differences. In fact, experiment 2 was conduct at a flow rate similar to the ones used for relative permeability measurements. Moreover, it is known that relative permeability values depend on saturation end-points (Ref.17).

Experiment 3 represents a drainage of perfluorinated hydrocarbon in a water-saturated plug. The forces regime analysis confirms a flooding condition as in case 2 . The sharp shape of the fronts is shown in FIG.4. As in experiment 3 we can observe that the FD simulator presents an excessively diffusive behaviour, especially for low saturation values.

Finally, a two-dimensional displacement experiment was performed and simulated. Deuterium water was injected in a real field sample, initially filled by water. For this case only a qualitative comparison has been carried out. FIG.5 shows NMR images at different times that confirm the permeability distribution obtained by a miniprobe: the obliquous dark layer in FIG.5 corresponds to a low permeability structure in a higher permeability matrix.

For the simulation only the centre of the core, excluding one centimetre on both sides, has been considered, since the resolution of the miniprobe doesn't consent to have reliable data in this zone.

Simulation results are shown in FIG.6 for the MFE-TVD FV and in FIG.7 for the FD methods. Both the numerical models are able to capture the presence of the low permeability structure. Nevertheless, the experimental front results in a more accentuated delay on the left side, which is probably related to the presence of a smaller low permeability structure. At the same time we underline that 
the FD simulator shows an unphysical front dispersion as an effect of the intrinsic numerical diffusion.

\section{CONCLUSIONS}

1) MRI is proving to be a reliable and powerful nondestructive tool for investigating the movement of fluids inside porous media. It makes it possible to identify heterogeneities and evaluate the effect of different regimes of forces to give realistic information on the recovery mechanisms at field scale.

2) The use of numerical tools at core scale makes it possible to integrate experimental methodologies to investigate porous media physics in a wide range of scenarios.

3) In particular, the coupling of MRI and numerical simulation makes it possible to validate experimental flow functions and facilitate the transfer of lab data to reservoir study.

4) MFE-TVD FV has proved to be a more accurate solution with respect to the FD simulator since it is not affected by numerical dispersion, which can cover the physical phenomena, especially at viscous regime.

5) The use of permeability maps from miniprobe make it possible to complete the unknown petrophysical parameters for a more reliable numerical core simulation.

\section{ACKNOWLEDGEMENTS}

We'd like to thank AGIP SpA management for the permission to publish the paper.

Particular thanks to $\mathrm{N}$. Bona and $\mathrm{O}$. Meazza for miniprobe data and, not least, E. Rossi for helpful discussion during the work.

\section{NOMENCLATURE}

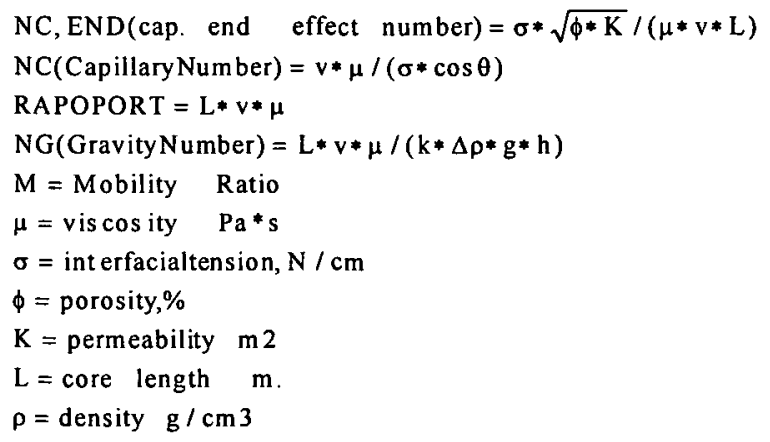

\section{REFERENCES}

1 Edelstein, W. A., H.J. Vinegar, P.N. Tutunjian, P.B. Roemer, and O.M. Mueller, "NMR Imaging for Core Analysis," Soc. Petr. Eng. Annual Tech. Conf., SPE 18272, Houston (Oct. 2-5, 1988).
2 Durlofsky L.J., "Accuracy of mixed and control volume finite element approximations to Darcy velocity and related quantities", Water Resources Research, 30, 965973 (1994)

3 Durlofsky L.J., Engquist B, Osher S., "Triangle based adaptive stencils for the solution of hyperbolic conservation laws", J. Comput. Phys., 98, 64 (1992)

4. Chardaire-Riviere, C., and J.C. Roussel, "Principle and Potential of Nuclear Magnetic Resonance Applied to the Study of Fluids in Porous Media," Revue de L'Istitut Francais Du Petrole, Vol.47, N², July-August 1992.

5 Mandava, S., C.M. Edwards, A.T. Watson, "NMR Imaging of Saturation During Immiscible Displacements," AIChE J., 36, 1680 (1990)

6 Chen, S., F. Qin, K.H. Kim, and A.T. Watson," NMR Imaging of Multiphase Flow in Porous Media," AIChE J., 39, 925 (1993).

7 Trangenstein J.A. and Bell J.B., "Mathematical structure of the Black-Oil model for petroleum reservoir simulation", SIAM J. Appl. Math., 49(3), 749-783, 1989.

8 Cominelli A., Consonni P., Mantica S., Manzini G., "High order mixed finite element TVD finite volume schemes for Black-Oil simulation", presented at this conference.

9 Brezzi F., J. Douglas, L.D. Marini,"Two Families of MFE for Second Order Elliptic Problems," Numer. Math. 47,217-235, 1985.

10 Bergamaschi L., Mantica S., Saleri F., "Mixed finite element approximation of Darcy's Law in porous media", CRS4 Technical Report \# 94/20

11 Dawson C., "Godunov-mixed methods for advectiondiffusion equations in multidimensions", SIAM J. Numer. Anal., 30(5), 1315-1332, 1993

12 Bell J.B., Colella P., Trangenstein J.A., "High order Godunov methods for general system of hyperbolic conservation laws", J. Comp. Phys., 82, 362-397, 1989

13 Ragazzini, G., C. Venturini, "A New Technique to Obtain the Real Capillary Pressure-Saturation Curve Directly From Centrifuge Experiments," Soc. Core Analysis, 14-16 Sep. (1992)

14 Oak, M.J.,"Three-Phase Relative Permeability of Water-Wet Berea," SPE/DOE 20183

15 Mohanty, K.K., A.E. Miller,"Factor Influencing Unsteady Relative Permeability os a Mixed-Wet Reservoir Rock," SPEFE, Sep. 1991, 349-358

16. Zhou D., F.J. Fayers, F.M. Orr Jr.,"Scaling of Multiphase Flow in Simple Heterogeneous Porous Media" SPE/DOE 27833

17 Honarpour, M., L. Koederitz, A. Herbert Harvey:Relative Permeability of Petroleum Reservoir, CRC Press, Boca Raton, Florida, 1987 Second Printing 
Tab. 1 - Samples petrophysical parameters and experimental flood conditions.

\begin{tabular}{|ccccc|}
\hline EXP. & $\begin{array}{c}\text { POROSITY } \\
(\%)\end{array}$ & $\begin{array}{c}\text { PERMEABILITY } \\
(\mathrm{mD})\end{array}$ & DISPLACEMENT & $\begin{array}{c}\text { FLOW RATE } \\
(\mathrm{ml} / \mathrm{min})\end{array}$ \\
\hline 1 (BEREA) & 21 & 850 & imbibition & 0.2 \\
2 (BEREA) & 21 & 850 & imbibition & 2 \\
3 (BEREA) & 18 & 880 & drainage & 1.5 \\
4 (RESER. & 8.7 & 12 & - & 0.1 \\
\hline
\end{tabular}

Tab.2 - Flow parameters.

\begin{tabular}{|ccccccc|}
\hline EXP & NC & NC,END & RAPOPORT & NG & $M$ & FLOW REGIME \\
\hline 1 & $1.35 E-07$ & $2.52 E+03$ & $4.11 E-01$ & $1.65 E+00$ & $1.00 E-03$ & CAPILLARY \\
2 & $1.35 E-06$ & $2.52 E+02$ & $4.11 E+00$ & $1.65 E+01$ & $1.00 E-03$ & VISC.-CAPIL. \\
3 & $3.66 E-06$ & $3.38 E+00$ & $7.53 E+00$ & $4.00 E+01$ & $5.63 E+00$ & VISC.-CAPIL. \\
4 & $5.60 E-03$ & $5.70 E-05$ & $3.40 E-01$ & $7.40 E+03$ & 1 & VISC. \\
\hline
\end{tabular}

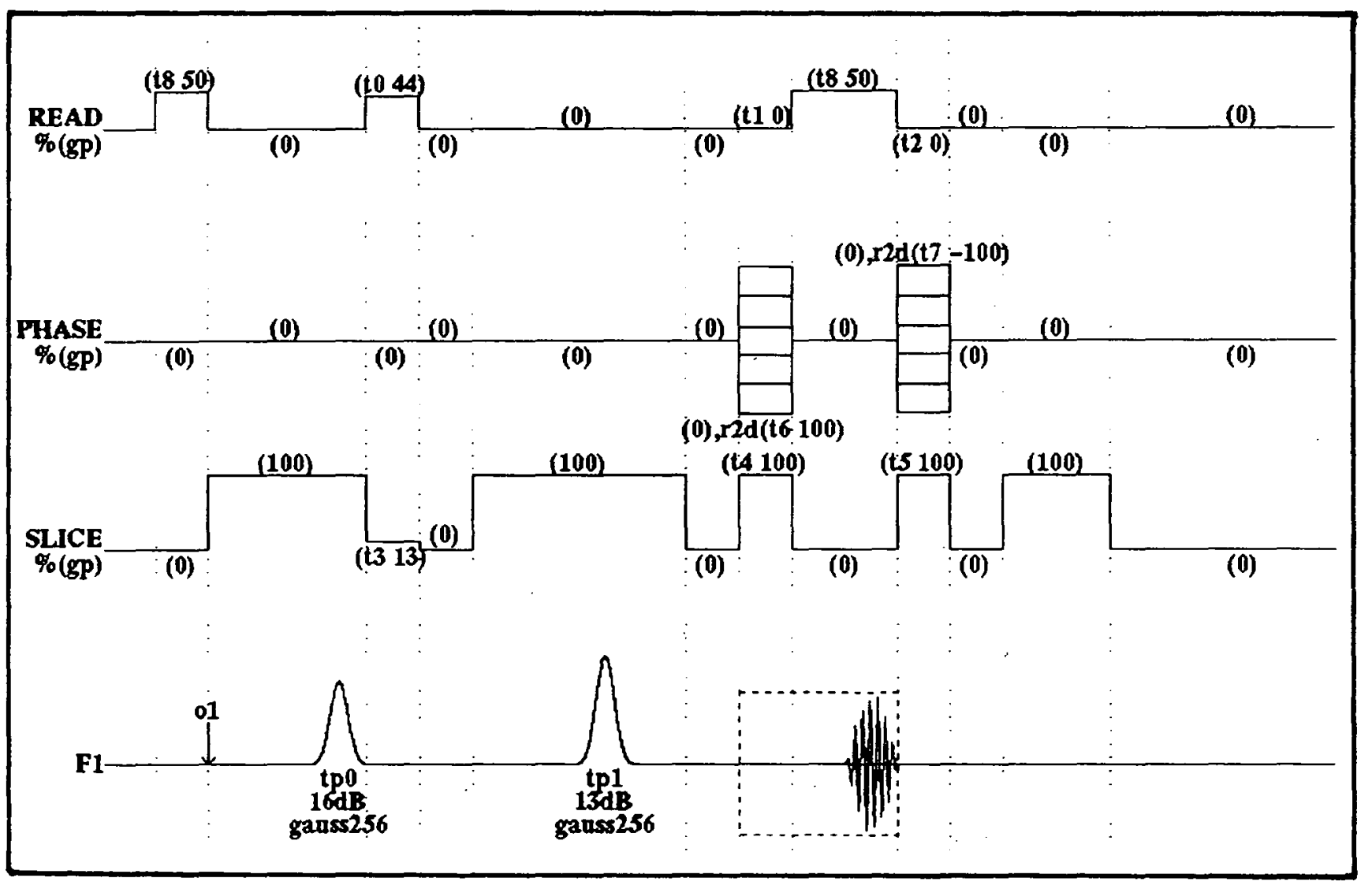

Fig. 1 - MRI Sequence used to acquire 1D and 2D images 

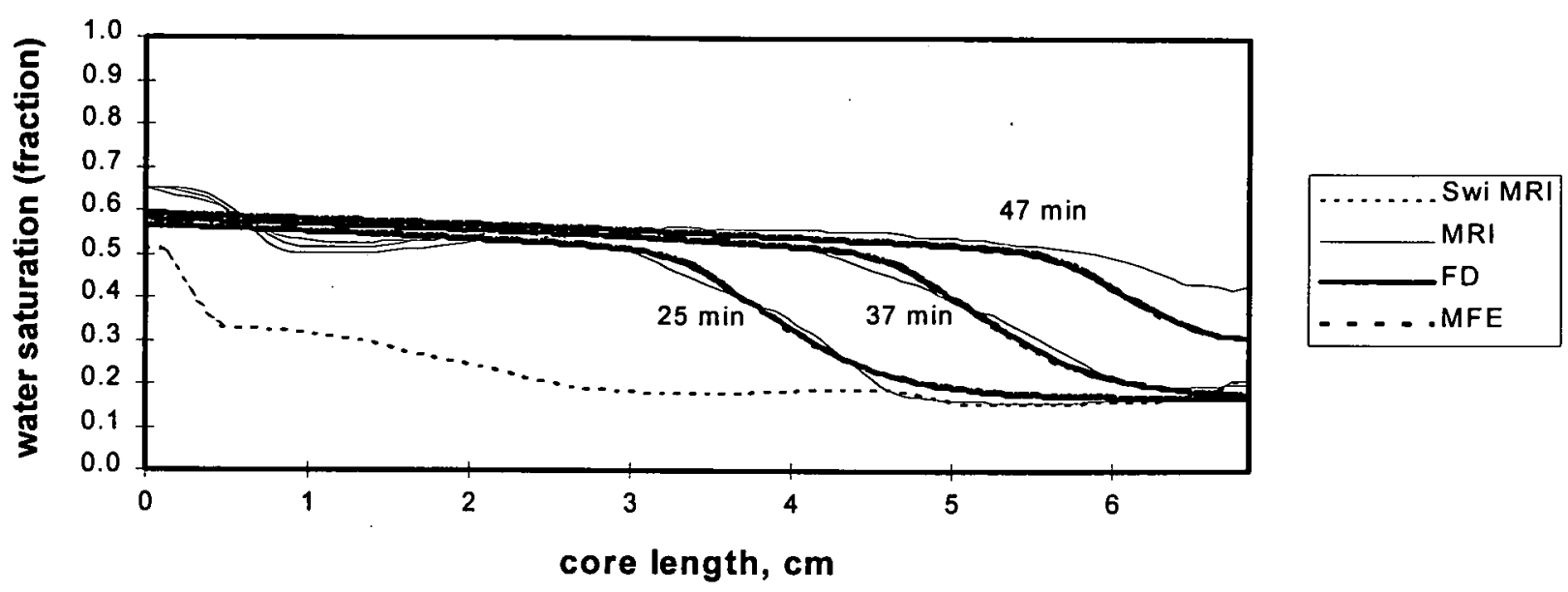

Fig. 2 - Experiment $\mathrm{n}^{\circ} 1$ : Comparison between experimental and simulated data.

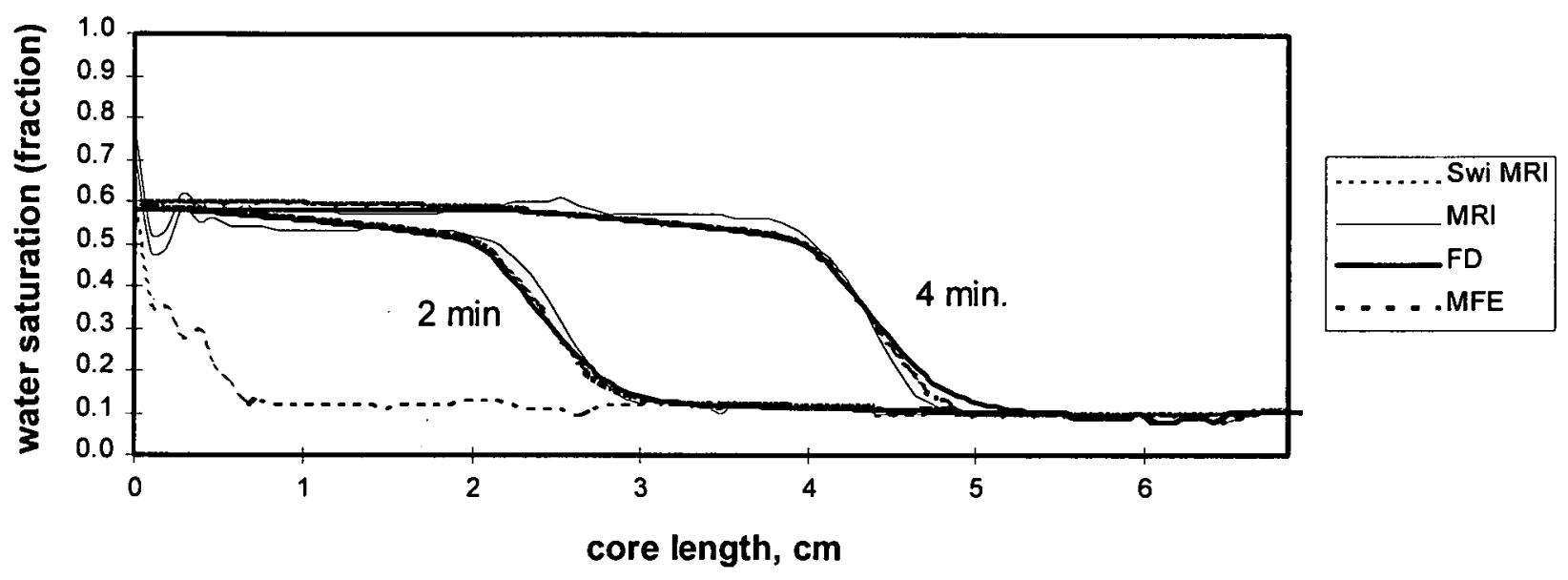

Fig. 3 - Experiment $n^{\circ} 2$ : Comparison between experimental and simulated data.

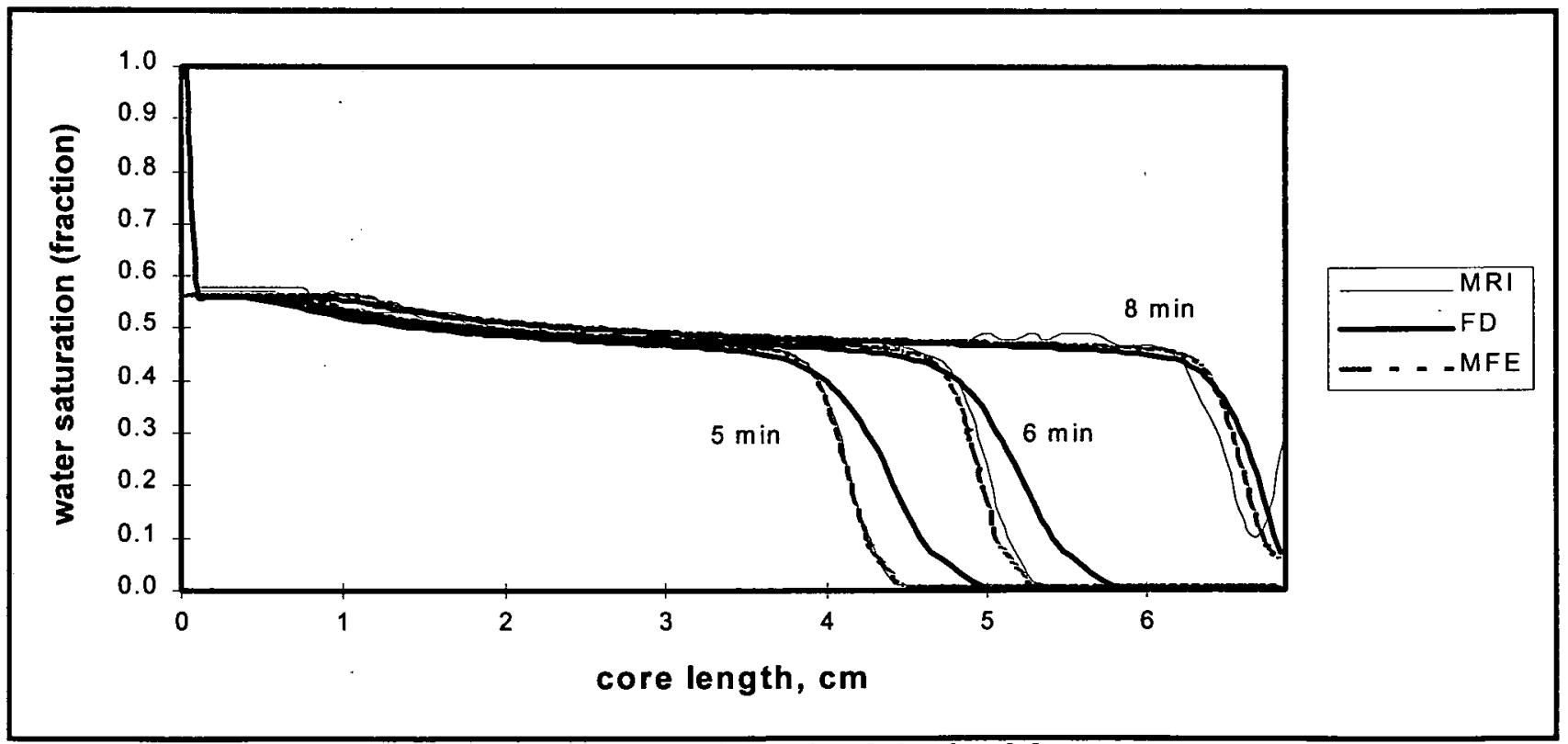

Fig. 4 - Experiment $n^{\circ}$ : Comparison between experimental and simulated data. 
The continuous white lines indicate the miniprobe matrix limit, the dashed line indicates the position of the front.
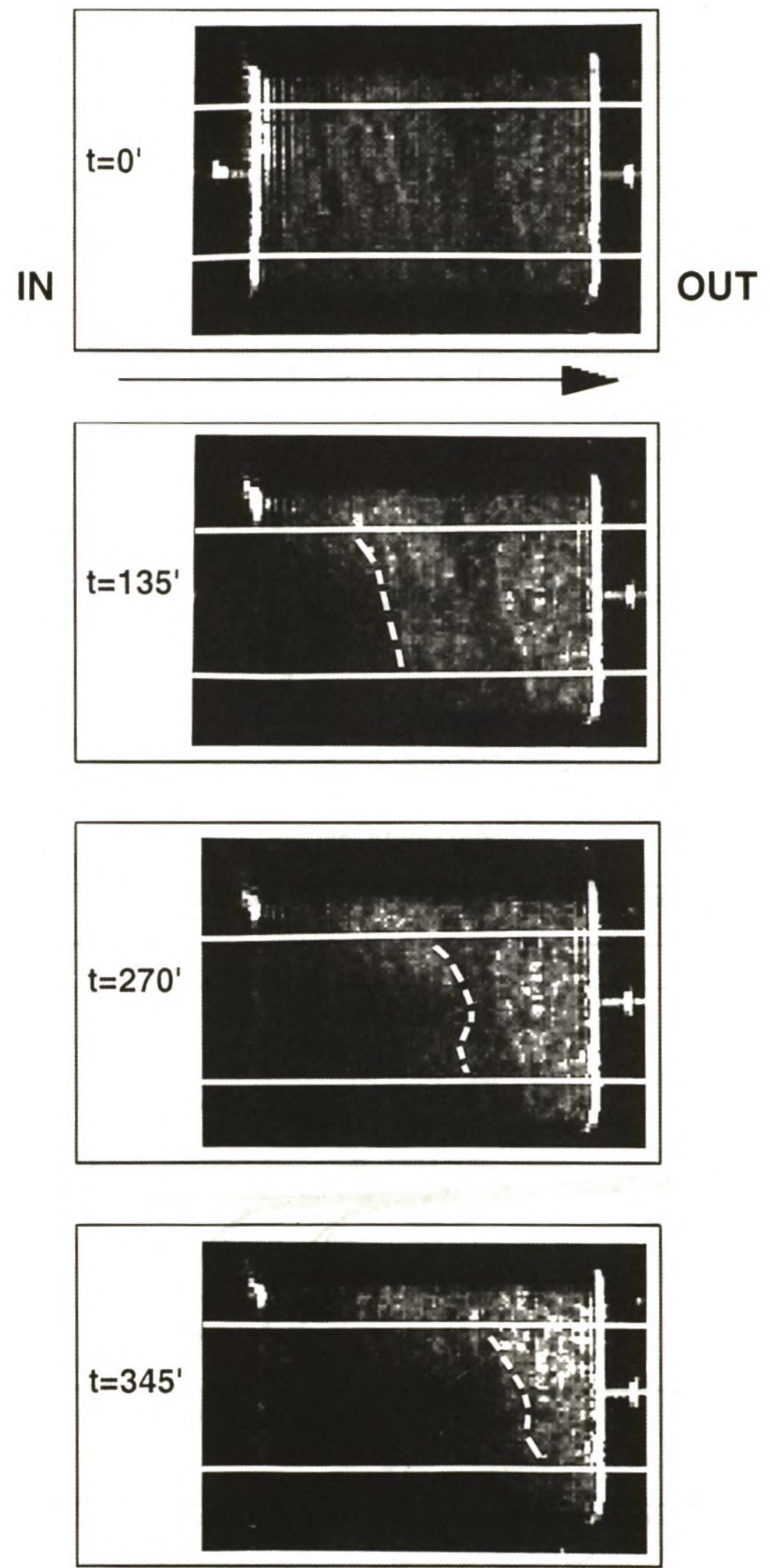

Fig. 5 - 2D experiment : injection of $\mathrm{D}_{2} \mathrm{O}$ in a water saturated sample ( $Q=0.1 \mathrm{cc} / \mathrm{min}$ ) - NMR Images 

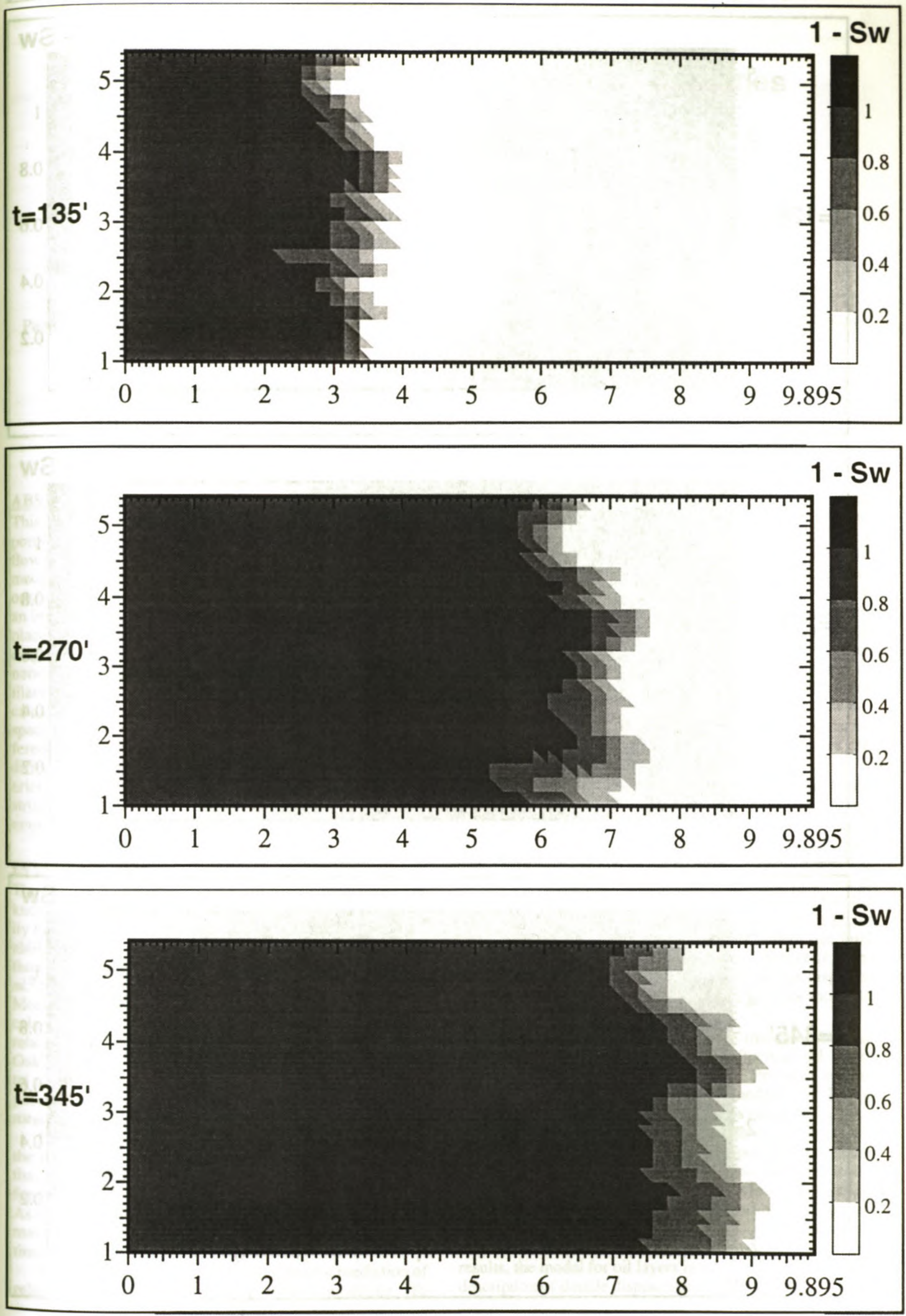

Fig. 6 - 2D experiment : MFE-TVD FV simulation 

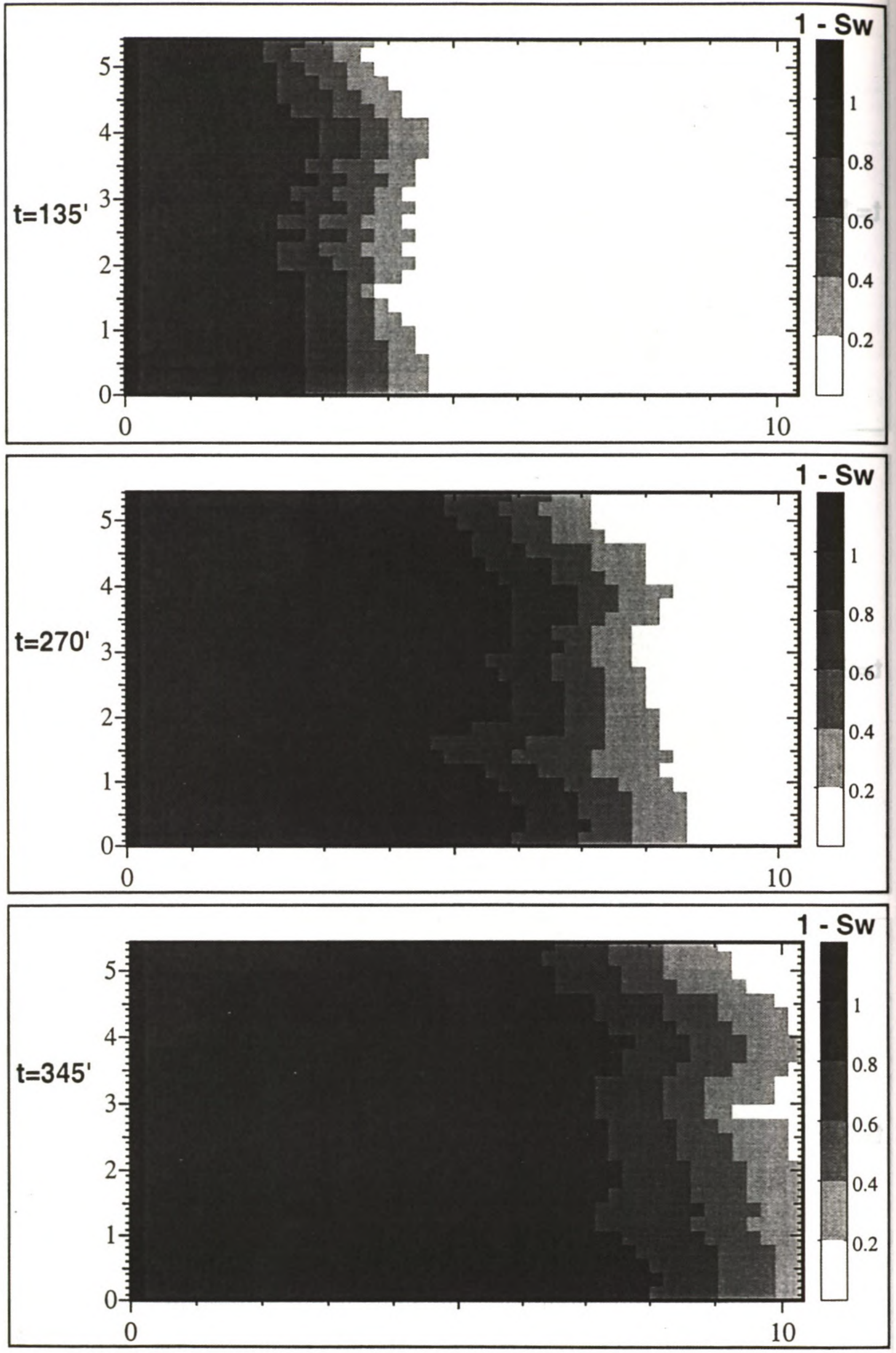

Fig. 7 - 2D experiment : standard FD simulation 